\title{
The Advantages of Network Data Transmission of Optical Fiber Data and its Principle
}

\author{
Tianle Dai
}

\author{
Nansha College Preparatory Academy \\ 2220021@ncpachina.org
}

\begin{abstract}
ABSTACT
Now China has popularized optical fiber. However, many people still do not know the advantages of them [1]. Optical fiber communication is the data and signals transmission using optical fiber as the medium. It can be used to transmit analog and digital signals. Taking optical fiber as the research object, this paper focuses on the principle of optical fiber data transmission. Also, this paper compares it with cooper wire data transmission, and demonstrates the multiple advantages of optical fiber data transmission.
\end{abstract}

Keywords: Optical fiber transmission principle, Optical fiber characteristic, Optical fiber advantage, Optical fiber transmission.

\section{INTRODUCTION}

The optical fiber is used to replace the copper wire for network transmission in many cities in China not long ago[1]. As far as the transmission rate is concerned, the transmission rate of the optical fiber is much faster than that of traditional copper wire. The reason behind this, however, is not known by everyone, and this paper intends to reveal to the audience the real reason why the transmission rate of optical fiber is faster than that of copper wire. Light travels a distance of 7.5 times around the Earth per second, which is considered as the reason why photo communication is faster than telecommunication through copper wires. It is completely wrong to think so. The speed referred to in communication is the ability to transmit data rather than the speed of signal transmission. The speed of signal transmission has not had much difference between an electrical signal conducted in a copper wire and an optical signal conducted in an optical fiber. However, at the same time, the amount of data transmitted through optical fiber communication is much larger than that of copper wire [2]. Therefore, the difference of optical fiber and cooper wire in data transmission rate is not influenced by the signal transmission speed. Given this, starting from the principle of optical fiber transmission, this paper will analyze its transmission characteristics, and explore its advantages in network data transmission. Then, this paper will further reveal that the transmission effect of optical fiber is better than that of copper wire.

\section{OPTICAL FIBER TRANSMISSION PRINCIPLE AND CHARACTERISTICS}

\subsection{Principle of optical fiber transmission}

The law of independent propagation of light holds that when light rays from different sources pass through a point in a medium in different directions, the rays do not affect each other, as if the other rays did not exist. (Figure 1)

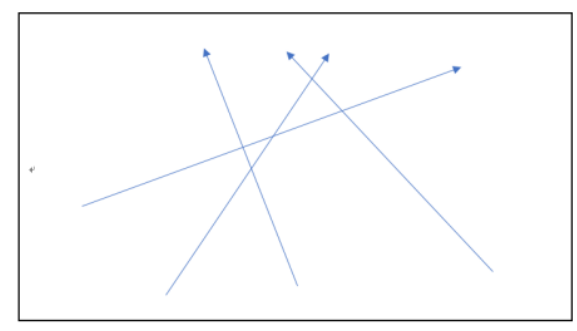

Figure 1 The law of independent propagation of light 
The laws of linear propagation and refraction and reflection of light hold that light travels in a straight line in an isotropic homogeneous medium (constant refractive index $n$ ). Light in the propagation of light encounters two different media smooth interface, light reflection and refraction. The speed of light propagation in a homogeneous medium is: $\mathrm{V}=\mathrm{c} / \mathrm{n}$, where $\mathrm{c}$ is the speed of light propagation in a vacuum; $n$ is the refractive index of the medium[3].

Due to the law of reflection, the reflected ray is in the plane determined by the incident ray and the normal, the reflection ray and the incident ray are on both sides of the normal, and the angle of reflection is equal to the angle of incidence[3].

The law of refraction is that the refraction ray is located in the plane determined by the incident ray and the normal, and the refraction ray and the incident ray are located on both sides of the normal[3].

In the process of light propagation, if the light propagates from a medium to another medium at the intersection, because the two mediums have different refractive indices, reflection and refraction will occur at the intersection. Generally, the refractive index of the medium with a larger refractive index is called the optically denser medium, and the small refractive index is called the optically thinner medium[3].

To make sure that the optical signal in the fiber can be transmitted over long distances, it is necessary to make the optical signal be reflected repeatedly and totally in the fiber in order to ensure minimum attenuation and dispersion to reach the far end. The two conditions for achieving total reflection are as follows: the refractive index $\mathrm{n} 1$ of the fiber core must be greater than the refractive index $\mathrm{N} 2$ of the fiber cladding; when the light entering the fiber incidents at the interface of the core and cladding, the angle of incidence should be greater than the critical angle.

\subsection{Characteristics of optical fiber transmission.}

Optical fiber transmission system mainly consists of a light source (also known as an optical transmitter), transmission medium, and detector (also known as optical receiver). In the optical fiber transmission between computer networks, the optical fiber transceiver finishes the work of the light source and detector[4]. This is a simple device to realize the connection between twisted pair and optical fiber, and can convert the signal transmitted by the twisted pair into a signal that can be transmitted through the optical fiber (optical signal). Optical fiber transmission is also bi-directional, and can convert the signal transmitted by optical fiber into the transmit signals in the twisted-pair cable to achieve data transmission between networks. In the normal transmission process of video, audio, data, etc., the optical transceiver finishes the work of the light source and detector, which is the device that turns multiple E1 signals into optical signals and transmits them, and the so-called $\mathrm{E} 1$ is the data transmission standard of trunk line[5].

The converted signal is divided into analog optical transmitter and receiver and digital optical transmitter and receiver. Therefore, the optical fiber transmission system can be divided into the digital transmission system and the analog transmission system based on the transmission signal. The analog transmission system is the analog modulation of light intensity, which changes the input signal into a continuous change of amplitude (frequency or phase) of the transmitted signal[5]. Digital transmission system converts the input signal into the "I" and "O" pulse signals, and uses it as the transmission signal, and then restores it to the original signal at the receiving end. Different optical fiber transmission signals require different equipment. Optical fiber as a transmission medium is a significant element in optical fiber transmission systems, which can be classified based on different ways. According to the mode of transmission, light is transmitted only along the inner core of the fiber. If only the main mode is transmitted, it's called SingleMode Fiber. The transmission of multiple modes in an optical fiber is called Multi-Mode Fiber.

According to the diameter, optical fiber is divided into the following categories: Step index fiber (SIF), Graded index fiber (GIF), Driver fiber (RIF), and W-type fiber[2].

\section{THE ADVANTAGES OF THE OPTICAL FIBER NETWORK DATA TRANSMISSION}

\subsection{The advantages of bandwidth of the optical fiber transmission}

The width of the frequency band reveals the size of the transmission capacity. The higher the frequency of the carrier wave is, the greater the bandwidth of the signal can be transmitted. In the VHF frequency band, the carrier frequency is $48.5 \mathrm{MHz}$ to $300 \mathrm{Mhz}$. The bandwidth is about $250 \mathrm{MHz}$, which can only transmit 27 sets of TV and dozens of FM broadcasts[6].

The frequency of optical light reaches $100000 \mathrm{GHz}$, more than one million times as high as the VHF band. Although the bandwidth is affected by the different losses of optical fiber for different frequencies of light, the bandwidth in the lowest loss area is also up to 30,000 $\mathrm{GHz}[6]$. 
The current bandwidth of a single light source accounts for only a small part of it (the frequency band of multi-mode fiber is about several hundred $\mathrm{MHz}$, and a good single-mode fiber can reach more than $10 \mathrm{GHz}$ ). Using advanced coherent optical communication can arrange 2,000 optical carriers in the range of $30,000 \mathrm{GHz}$ for wavelength division multiplexing, which can accommodate millions of channels [6].

\subsection{Advantages of the low loss of the optical fiber transmission}

In a system of coaxial cables, even the best cables will lose more than $40 \mathrm{~dB}$ per kilometer when transmitting 800 $\mathrm{MHz}$ signals. In contrast, the optical fiber will lose much less[6].

The transmission of 1.31 um light has a loss of $0.35 \mathrm{~dB}$ per kilometer or less. If 1.55 um light is transmitted, the loss per kilometer is even less, up to $0.2 \mathrm{~dB}$ or less, which is 100 million times smaller than the power loss of coaxial cable, so that it can transmit much farther[7]. For this reason, optical fibers cost less energy since the loss during transmission is very low comparing to copper wires.

In addition, the optical fiber transmission loss has two other characters. One is that it has the same loss in all cable channels and does not require the introduction of equalizers for equalization as cable trunks do, and the other characteristic is that its loss hardly varies with temperature, so you do not have to worry about fluctuations in the level of the trunk due to changes in ambient temperature. This way, optical fiber is more reliable than copper wire.

\subsection{The advantages of easy transportation and easy installation of optical fiber}

The diameter of bare copper wire is $0.51 \mathrm{~mm}$, and the core is 8 cores and 4 pairs of twisted pairs[3]. The glass fiber can replace copper wire to transmit data because of the characteristics of light, which has small specific gravity and has the characteristics of small diameter and light weight. Therefore, the diameter of the single-mode fiber core is generally $4 \mathrm{um} \sim 10 \mathrm{um}$, and the outer diameter is only $125 \mathrm{um}$. With the waterproof layer, reinforcement, sheath, etc., the diameter of the optical cable composed of 4 to 48 fibers is less than $13 \mathrm{~mm}$, which is much smaller than the diameter of a standard copper cable. This shows that the use of light can reduce a lot of pressure on cable transport. At the same time, these features also make the installation of optical fiber pairs more convenient compared to copper wire[7].

\subsection{The optical fiber transmission has a strong anti-interference ability}

Since the basic component of an optical fiber, quartz, is not conductive, electromagnetic fields cannot influence optical fiber transmission. While copper wires will be hugely affected by surrounding electromagnetic fields. What make things worse is that copper wires can not only being interference by electromagnetic field, but also being malicious snooped.

Therefore, comparing to copper wire, fiber optic transmission has a stronger ability to resist electromagnetic interference, and industrial interference.

Also, signals transmitted in the optical fiber are less susceptible to eavesdropping, thus facilitating confidentiality. Making optical fiber data transmission more stable in some special situations.

\subsection{The fiber optic transmission has high fidelity}

Since the relay amplification is not needed in the optical fiber transmission, no new nonlinear distortion is introduced by the amplification. As long as the linearity of the laser is good, it can transmit the TV signal with high fidelity[8].

Real-world experiments show that a good AM fiber optic system carrier combination of C/CTB is more than $70 \mathrm{~dB}$, and the $\mathrm{cM}$ is also more than $60 \mathrm{~dB}$, much higher than the general cable trunking system of nonlinear distortion indicators [6].

\subsection{Conclusion of advantages of optical fiber in data transmission}

As the number of Internet users increases, the demand for bandwidth increases [2]. The low bandwidth provided by copper wire can't fulfill people's needs. Optical fiber, instead, can provide up to 40 times more bandwidth in a single fiber than a copper wire. While optical fibers also take less space than copper wire that there is a huge difference in data transmission rate between optical fiber and copper wire of the same thickness. The difference between bandwidths is the main reason why people replace the copper wire with optical fiber on large-scale data transmission. To make their difference even bigger, optical fiber has very low signal loss through longdistance data transmission. Network providers can hugely improve the quality of their services by replacing copper wires with optical fibers [4]. In addition to all the advantages of optical fiber in data transmission, optical fibers can be easier installed comparing to copper wires. 


\section{THE REASON WHY COPPER WIRE HAS NOT BEEN COMPLETELY REPLACED BY OPTICAL FIBER}

Since optical fiber is so good, why copper wires are still existing today? This is because copper wires have some characteristics that optical fibers do not have. Copper wires are made of copper so besides data transmission, it can also transmit electricity. So copper wire is still critical in a small number of scenarios that require the transmission of signals and power supply [1]. Even if optical fiber is very practical in large-scale transmission, for general users, the current copper wire can fully meet the needs of data transmission rate and stability. Since a normal personal user doesn't have the need of huge bandwidth, ultra-low signal loss rate or strong anti-interference that optical fiber provides. For this reason, most household Internet devices in a short period will not have the optical fiber interface.

\section{CONCLUSION}

Nowadays, the popularity of optical fiber makes the traditional copper wire in signal transmission has become more and more disadvantaged. In conclusion, the optical fiber transmission has the advantages of low attenuation, wide frequency band, strong anti-interference, high safety performance, small size and light weight, which make it is a superb choice in long-distance transmission and special environment, etc. But copper wires are still not fully replaced by optical fibers since copper wires can still needs of data transmission rate and stability.

\section{REFERENCES}

[1] Wang Ruijun. Discussion on the development status and trend of the optical fiber data transmission technology in the new era [J]. Digital Communication World,2019(02):62.

[2] Meng Xiaodong, Wei Lihong,Feng Feng. Research on optimal data transmission path search mechanism for high-speed optical fiber communication networks[J]. Laser Journal,2020,41(10):197-200.

[3] Ke Youqiang, Song Wensheng. Multi-site hydroacoustic signal optical fiber transmission processing technology $[\mathrm{J}]$. Ship Electronics Engineering,2020,40(08):162-165.

[4] Wang Peibao. Application of the optical fiber transmission and digitalization in the image monitoring of substation $[\mathrm{J}]$. Science and Technology Innovation and Application,2021(02):179-181.
[5] Han Guoliang. The application of the optical fiber transmission technology in the design of cable television network $[\mathrm{J}]$. Western Radio and Television,2018(18):241-242.

[6] Zhang Jiwei. The development status and trend of the optical fiber data transmission technology in the new era[J]. Digital technology and application,2018,36(08):9+11.

[7] Liu Xiang, Yuan Ziqiao,Du Li,Chen Yinxiang. A multi-board pulse synchronization technique based on the optical fiber data transmission implementation $[\mathrm{J}]$. Fire Control Radar Technology,2018,47(02):52-54+62.

[8] Kong Wenqing,Li Zihang,Song Wanjie. Research on data processing and timing control in multiplexed optical fiber transmission[J]. Electronic Science and Technology,2018,31(05):1-4+15. 